\title{
Joint Space-Time Trellis Decoding and Channel Estimation in Correlated Fading Channels
}

\author{
Van Khanh Nguyen and Langford B. White, Senior Member, IEEE
}

\begin{abstract}
This letter addresses the issue of joint space-time trellis decoding and channel estimation in time-varying fading channels that are spatially and temporally correlated. A recursive space-time receiver which incorporates per-survivor processing (PSP) and Kalman filtering into the Viterbi algorithm is proposed. This approach generalizes existing work to the correlated fading channel case. The channel time-evolution is modeled by a multichannel autoregressive process, and a bank of Kalman filters is used to track the channel variations. Computer simulation results show that a performance close to the maximum likelihood receiver with perfect channel state information (CSI) can be obtained. The effects of the spatial correlation on the performance of a receiver that assumes independent fading channels are examined.
\end{abstract}

Index Terms-Diversity, Kalman filtering, multiple transmit and receive antenna, per-survivor processing, space-time trellis codes.

\section{INTRODUCTION}

$\mathbf{M}$ ETHODS for joint channel estimation and space-time trellis decoding in time-varying fading channels have been studied in [1]-[4]. These works however are based on the assumption of independent and identically distributed (i.i.d) fading channels. In real propagation environment, the fades are not independent [5]. Spatially independent fades occur if the multipath reflections are uniformly distributed around the receiver and that the multiple antennas at the receiver are sufficiently spaced apart. This assumption is often hard to satisfy in practice due to the lack of local scatterrers around the base station and the limited size of the mobile unit. As a result, spatial correlation arises. Such spatial correlation can significantly reduce the performance of the receiver if it is not properly incorporated into the design of the receiver.

In this letter, we propose a per-survivor processing (PSP) receiver that performs joint space-time trellis decoding and channel estimation in spatially correlated time-varying fading channels. This proposed PSP receiver incorporates the per-survivor processing and Kalman filtering into the Viterbi algorithm similar to that in [4]. However, the proposed PSP receiver takes into account the channel estimation errors when computing the path metric in the Viterbi algorithm while the PSP receiver in [4] computes the path metric as if there is no channel

Manuscript received September 8, 2003; revised November 29, 2003. The associate editor coordinating the review of this manuscript and approving it for publication was Prof. Zhi Ding.

V. K. Nguyen is with the School of Engineering and Technology, Deakin University, Geelong, Vic. 3217, Australia (e-mail: vknguyen@ deakin.edu.au; vknguyen@eleceng.adelaide.edu.au).

L. B. White is with the Department of Electrical and Electronic Engineering, The University of Adelaide, SA 5005, Australia (e-mail: Lang.White@ adelaide.edu.au).

Digital Object Identifier 10.1109/LSP.2004.830122 estimation error. These channel estimation errors are given by the Kalman filters. More importantly, we do not assume i.i.d fading channels and allow the channels to be spatially and temporally correlated. The proposed PSP receiver accounts for such spatio-temporal correlation in the channels when performing the channel estimation and the decoding steps. To our knowledge, there is no other work on receiver structure design which performs joint space-time trellis decoding and channel estimation in spatially correlated time-varying fading channels.

The following notation is used throughout the letter: The superscript ${ }^{T}$, ${ }^{*}$ and ${ }^{H}$ denote the transpose, complex conjugate and conjugate transpose operations, respectively. The symbols $\otimes$ and $\delta()$ denote the Kronecker product operation and the Dirac delta function, respectively. The matrix $\mathbf{I}_{N}$ is the $N \times N$ identity matrix.

\section{SYSTEM MODEL}

Consider a wireless communication system with $n_{T}$ transmit and $n_{R}$ receive antennas. At each time $t$, the encoder produces $n_{T}$ outputs $x_{1}(t), x_{2}(t), \ldots, x_{n_{T}}(t)$ where $x_{i}(t)$ is a signal from a certain signal constellation with unit average energy. The $n_{T}$ coded symbols output from the encoder are then simultaneously transmitted by $n_{T}$ antennas. At the receiver, the received signal at the $j$ th receive antenna is

$$
\begin{aligned}
r_{j}(t) & =\sum_{i=1}^{n_{T}} h_{i, j}(t) x_{i}(t) \sqrt{E_{s}}+n_{j}(t), \\
& =\sqrt{E_{s}} \mathbf{x}(t) \mathbf{h}_{j}(t)+n_{j}(t)
\end{aligned}
$$

where $E_{s}$ is the transmitted power per symbol at each transmit antenna, $\mathbf{x}(t)=\left[\begin{array}{llll}x_{1}(t) & x_{2}(t) & \cdots & x_{n_{T}}(t)\end{array}\right]$, $\mathbf{h}_{j}(t)=\left[\begin{array}{llll}h_{1, j}(t) & h_{2, j}(t) & \cdots & h_{n_{T}, j}(t)\end{array}\right]^{T}$ and $n_{j}(t)$ are independent samples of a zero-mean complex Gaussian process with variance $N_{0}$. It is further assumed that the additive noise is white in space and time. The coefficient $h_{i, j}(t)$ is the fading gain for the path from transmit antenna $i$ to receive antenna $j$. The gain $h_{i, j}(t)$ is modeled as a complex Gaussian random process with zero-mean and variance one. Stacking the observations from the receive antennas yields the $n_{R}$ sufficient statistics

$$
\begin{aligned}
\mathbf{r}(t) & =\left[r_{1}(t) r_{2}(t) \cdots r_{n_{R}}(t)\right]^{T} \\
& =\sqrt{E_{s}} \mathcal{X}(t) \mathbf{h}(t)+\mathbf{n}(t)
\end{aligned}
$$

where $\mathbf{h}(t)=\left[\mathbf{h}_{1}^{T}(t) \mathbf{h}_{2}^{T}(t) \cdots \mathbf{h}_{n_{R}}^{T}(t)\right]^{T}, \mathbf{n}(t)=$ $\left[n_{1}(t) n_{2}(t) \cdots n_{n_{R}}(t)\right]^{T}$ and $\mathcal{X}(t)=\mathbf{I}_{n_{R}} \otimes \mathbf{x}(t)$. 


\section{Channel Model}

It has been shown in [7] that the time varying Rayleigh fading channels can be well described by a hidden Markov model. As has been done in [8], [9] for channel equalization problems, the time varying fading channels can be modeled by the multichannel AR process of order $P$

$$
\mathbf{h}(t)=\sum_{p=1}^{P} \mathbf{A}(p) \mathbf{h}(t-p)+\mathbf{v}(t)
$$

where the matrices $\mathbf{A}(p)$ are the time invariant matrices which are determined from the second-order statistic of the fading channels and $\mathbf{v}(t)$ is a zero mean complex white Gaussian noise process with the correlation matrix $E\left[\mathbf{v}(t) \mathbf{v}^{H}(t+\tau)\right]=\mathbf{Q} \delta(\tau)$. By postmultiplying (3) with $\mathbf{h}^{H}(t-\tau)$ and taking the expectation on both sides we arrive at

$$
\mathbf{R}(-\tau)=\sum_{p=1}^{P} \mathbf{A}(p) \mathbf{R}(p-\tau)+\mathbf{Q} \delta(\tau), \quad \tau=0, \ldots, P
$$

where $\mathbf{R}(\tau)=E\left[\mathbf{h}(t) \mathbf{h}^{H}(t+\tau)\right]$ is the cross-correlation matrix. The matrices $\mathbf{A}(p)$ and $\mathbf{Q}$ are then obtained by solving the above equations. Due to the arrangement of $\mathbf{h}(t)$ in (2), $[\mathbf{R}(\tau)]_{e, f}$ represents the cross-correlation between the two fading gains $h_{p, l}(t)$ and $h_{q, m}(t+\tau)$ where $1 \leq p, q \leq n_{T}$; $1 \leq l, m \leq n_{R} ; e=p+(l-1) n_{T}$ and $f=q+(m-1) n_{T}$. We use the physical scattering model in [5] to determine the cross-correlation matrices $\mathbf{R}(\tau)$ and use these matrices to solve (4) for a specified $P$ to obtain an AR approximation to this covariance. This space-time cross-correlation is given in [5] as

$$
\begin{aligned}
& {[\mathbf{R}(\tau)]_{e, f}=E\left[h_{p, l}(t) h_{q, m}^{*}(t+\tau)\right] } \\
&=\frac{\exp \left[j c_{p q} \cos \left(\alpha_{p q}\right)\right]}{I_{0}(\kappa)} \\
& \times I_{0}\left(\left\{\kappa^{2}-a^{2}-b_{l m}^{2}-c_{p q}^{2} \Delta^{2} \sin ^{2}\left(\alpha_{p q}\right)\right.\right. \\
& \quad+2 a b_{l m} \cos \left(\beta_{l m}-\gamma\right) \\
& \quad+2 c_{p q} \Delta \sin \left(\alpha_{p q}\right) \\
& \times\left[a \sin (\gamma)-b_{l m} \sin \left(\beta_{l m}\right)\right] \\
&-j 2 \kappa\left[a \cos (\mu-\gamma)-b_{l m} \cos \left(\mu-\beta_{l m}\right)\right. \\
&\left.\left.\left.-c_{p q} \Delta \sin \left(\alpha_{p q}\right) \sin (\mu)\right]\right\}^{1 / 2}\right)
\end{aligned}
$$

where $a=2 \pi f_{D} \tau ; b_{l m}=2 \pi d_{l m} / \lambda ; c_{p q}=2 \pi \delta_{p q} / \lambda ; \lambda$ is the carrier wavelength; $f_{D}$ is the Doppler shift; $\mu$ is the mean of the angle of arrival (AOA) at receiver; $\kappa$ controls the spread of the AOA; and $\gamma$ is the direction of motion of the receiver. Other geometric parameters are defined in Fig. 1 where $T X_{p}, T X_{q}$, $R X_{l}$ and $R X_{m}$ denote transmit antenna $p$, transmit antenna $q$, receive antenna $l$ and receive antenna $m$, respectively.

The proposed receiver in this letter employs Kalman filters for estimating the fading channel. Thus, in order to keep the complexity of the receiver at a reasonably level, we use the first order AR process to model the fading channel. For $P=1$, the channel fading process has an AR representation

$$
\mathbf{h}(t)=\mathbf{A h}(t-1)+\mathbf{v}(t) .
$$

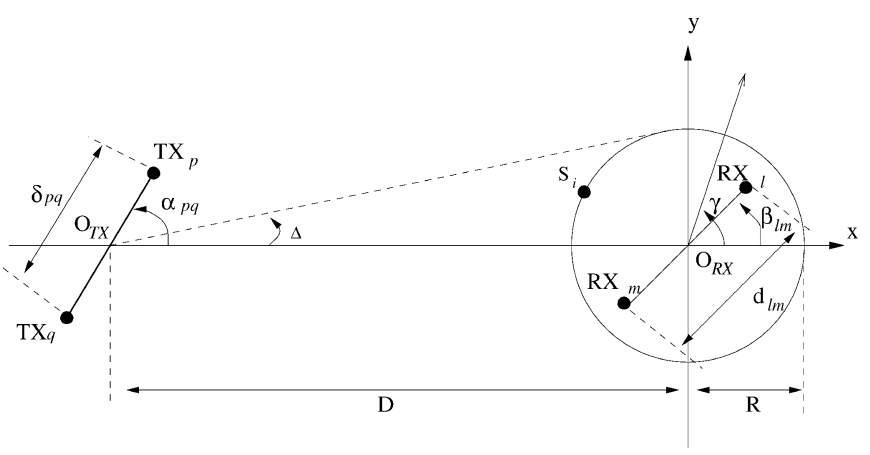

Fig. 1. Channel model with local scaterrers $S_{i}$ around the receiver.

The matrices $\mathbf{A}$ and $\mathbf{Q}$ are evaluated by solving (4) for $\tau=0,1$

$$
\begin{aligned}
& \mathbf{A}=\mathbf{R}(-1) \mathbf{R}^{-1}(0) \\
& \mathbf{Q}=\mathbf{R}(0)-\mathbf{A R}(1)
\end{aligned}
$$

where $\mathbf{R}(0)$ and $\mathbf{R}(1)$ are defined in (5).

\section{PSP RECEIVER}

In recent years, per-survivor processing [10] with Kalman filtering has been seen as an attractive approach to performing maximum likelihood sequence estimation over mobile radio channels that are rapidly time-varying [8] (with $n_{T}=n_{R}=1$ ). In [6], we have extended this method to perform joint channel estimation and decoding of block codes in general diversity time-varying correlated fading channels. In this letter, we extend this work further to perform joint channel estimation and space-time trellis decoding by incorporating the per-survivor processing and Kalman filtering into the Viterbi algorithm. This receiver requires only one Kalman filter for each state in the trellis.

Let $S$ denotes the total number of states in the trellis. This PSP receiver works as follow: At the end of the symbol interval $t-1$, we assume that each state, $\mu_{s}(t-1)$ for $s=1, \ldots, S$, retains a survivor path. Associated with this survivor path are the channel estimate $\hat{\mathbf{h}}_{\mu_{s}}(t-1 \mid t-1)$ and the cumulative $\log$ likelihood path metric $\Gamma_{\mu_{s}}(t-1)$. At the $t$ th symbol interval, for all valid transitions to state $\mu_{y}(t), \mu_{s}(t-1) \rightarrow \mu_{y}(t)$, the following branch metric is computed:

$$
\begin{aligned}
\Lambda\left(\mu_{s}(t-1)\right. & \left.\rightarrow \mu_{y}(t)\right) \\
& =-\boldsymbol{\epsilon}_{s, y}^{H}(t) \boldsymbol{\Omega}_{s, y}^{-1}(t) \boldsymbol{\epsilon}_{s, y}(t)-\log \operatorname{det} \boldsymbol{\Omega}_{s, y}(t)
\end{aligned}
$$

where

$$
\begin{aligned}
\boldsymbol{\epsilon}_{s, y}(t) & =\mathbf{r}(t)-\sqrt{E_{s}} \mathcal{X}_{s, y}(t) \hat{\mathbf{h}}_{\mu_{s}}(t \mid t-1), \\
\boldsymbol{\Omega}_{s, y}(t) & =E_{s} \mathcal{X}_{s, y}(t) \boldsymbol{\Sigma}_{\mu_{s}}(t \mid t-1) \mathcal{X}_{s, y}^{H}(t)+N_{0} I_{n_{R}}
\end{aligned}
$$

and $\mathcal{X}_{s, y}(t)$ are the coded symbols that are output by the encoder during the transition $\mu_{s}(t-1) \rightarrow \mu_{y}(t)$. This branch metric is different to the one used in [4] as it takes into account the channel estimation errors $\Sigma_{\mu_{s}}(t \mid t-1)$. The predicted channel estimate $\hat{\mathbf{h}}_{\mu_{s}}(t \mid t-1)$ and its error covariance are computed using the time update equations

$$
\begin{aligned}
\hat{\mathbf{h}}_{\mu_{s}}(t \mid t-1) & =\mathbf{A} \hat{\mathbf{h}}_{\mu_{s}}(t-1 \mid t-1) \\
\Sigma_{\mu_{s}}(t \mid t-1) & =\mathbf{A} \Sigma_{\mu_{s}}(t-1 \mid t-1) \mathbf{A}^{H}+\mathbf{Q} .
\end{aligned}
$$




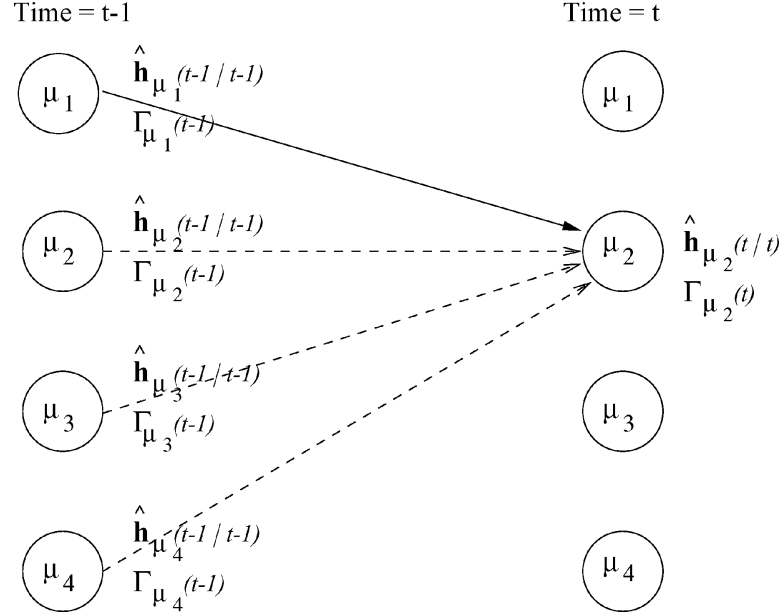

Fig. 2. PSP receiver with Viterbi algorithm.

Once the branch metric of all valid transitions $\mu_{s}(t-1) \rightarrow \mu_{y}(t)$ is computed, the survivor path sequence to the state $\mu_{y}(t)$ and its cumulative path metric $\Gamma_{\mu_{y}}(t)$ are determined by performing a maximization over the previous valid states $\mu_{s}(t-1)$

$$
\Gamma_{\mu_{y}}(t)=\max _{\mu_{s}(t-1)}\left[\Gamma_{\mu_{s}}(t-1)+\Lambda\left(\mu_{s}(t-1) \rightarrow \mu_{y}(t)\right)\right] .
$$

The channel estimate associate with this survivor path is then updated by a Kalman filter tuned to the coded symbols for the survivor transition. Let the predecessor state of $\mu_{y}(t)$ which maximizes the cumulative path metric $\Gamma_{\mu_{y}}(t)$ be denoted by $\mu_{\text {opt }}(t-1)$. The channel estimate at time $t$ of the survivor path at state $\mu_{y}(t)$ can be estimated by the Kalman filter tuned to $\mathcal{X}_{o p t, y}(t)$, the coded symbols for the survivor transition $\mu_{o p t}(t-$ 1) $\rightarrow \mu_{y}(t)$

$$
\begin{aligned}
\hat{\mathbf{h}}_{y}(t \mid t) & =\hat{\mathbf{h}}_{\text {opt }}(t \mid t-1)+\mathbf{G}_{o p t, y}(t) \epsilon_{o p t, y}(t), \\
\mathbf{G}_{o p t, y}(t)= & \sqrt{E_{s}} \boldsymbol{\Sigma}_{o p t}(t \mid t-1) \mathcal{X}_{o p t, y}^{H}(t) \Omega_{o p t, y}^{-1}(t), \\
\boldsymbol{\Sigma}_{y}(t \mid t)= & \boldsymbol{\Sigma}_{o p t}(t \mid t-1) \\
& \quad-\sqrt{E_{s}} \mathbf{G}_{o p t, y}(t) \mathcal{X}_{o p t, y}(t) \boldsymbol{\Sigma}_{o p t}(t \mid t-1)
\end{aligned}
$$

where $\epsilon_{o p t, y}(t)$ and $\Omega_{o p t, y}(t)$ are defined in (10). A pictorial description of the per-survivor processing with Viterbi algorithm is given in Fig. 2, assuming the 4 states space-time trellis codes with the rate of $2 \mathrm{bits} / \mathrm{s} / \mathrm{Hz}$.

\section{Performance Evaluation}

The performance of the PSP receiver proposed in this letter is evaluated by using computer simulations. We consider the down-link of a single user system where the BS is equipped with two transmit antennas and the MU has two receive antennas. The four-states QPSK space-time trellis codes in [11] is adopted. This space-time code achieves a bandwidth efficiency of $2 \mathrm{bits} / \mathrm{s} / \mathrm{Hz}$. We assume information is transmitted at the rate of $20 \mathrm{Ksps}$ at the carrier frequency of $2.4 \mathrm{GHz}$. The information is transmitted in frame of 25 coded symbols per transmit antenna. At the beginning of each frame, each antenna transmits an orthogonal sequence (with length of 2 symbols). These orthogonal pilot sequences are used by the receiver to obtain the
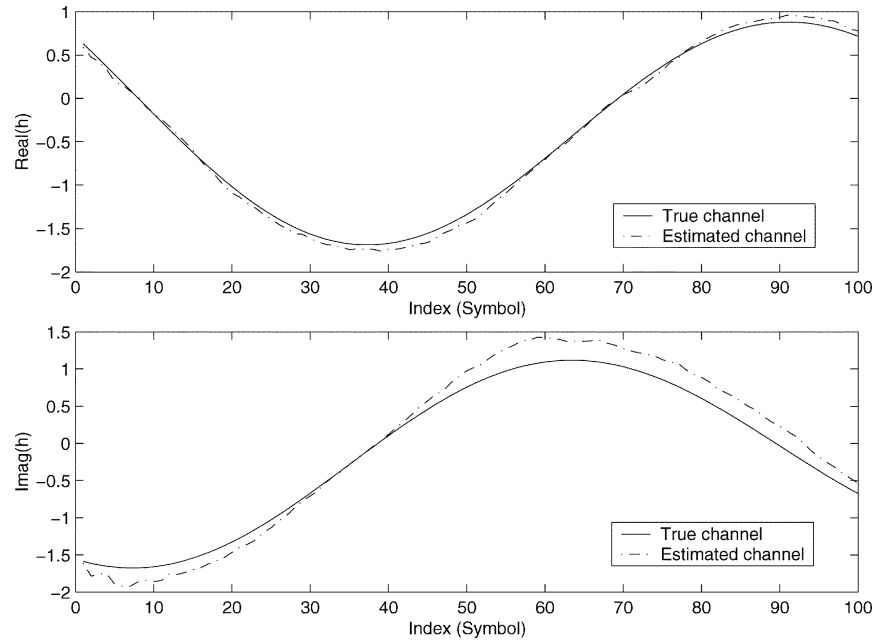

Fig. 3. True channel and KF based estimated channel.

initial fading channel estimate to initialize the Kalman filters. The spatially correlated time-varying fading channels with the space-time correlation (5) are generated based on the following physical parameters: The transmit antennas at the BS are separated by a distance of $10 \lambda$ while the receive antennas of the MU are separated by $0.5 \lambda$. The MU is moving in the direction $\gamma=45^{\circ}$. For the macrocells in urban, surburban and rural areas, the angular spread $\Delta$ at the BS is often less than $15^{\circ}$ and in some cases less than $5^{\circ}$. We choose $\Delta=10^{\circ}$ in this simulation. The angle spread for the $\mathrm{MU}$ is set to $2 / \sqrt{\kappa} \approx 66^{\circ}$ (i.e., $\kappa=3$ ) with the mean AOA $\mu=180^{\circ}$. The antenna array at the BS is assumed to be in parallel position to the MU antenna array (i.e., $\alpha_{1,2}=90^{\circ}, \beta_{1,2}=90^{\circ}$ ).

The matrices $\mathbf{A}$ and $\mathbf{Q}$ are determined using (7) and (8), respectively, where $\mathbf{R}(0), \mathbf{R}(-1)$ and $\mathbf{R}(1)$ are given by (5) using the above physical parameters. These correlation matrices can also be estimated directly from the received signals at the receiver using the technique described in [9]. In this letter we assume that these matrices are perfectly known at the receiver and focus on the problem of joint decoding and channel tracking. Fig. 3 shows how well the proposed PSP receiver tracks the channel variation over 100 symbols (i.e., 4 frames). We also compare the performance of the proposed PSP receiver with the maximum likelihood (ML) receiver that has perfect channel state information (CSI). Fig. 4 shows the frame error rate (FER) of these receivers at the fading rates of $f_{D} T_{s}=0.001$ and $f_{D} T_{s}=0.01$. At the fading rate of $f_{D} T_{s}=0.001$, the proposed PSP receiver is within $0.8 \mathrm{~dB}$ of the ML receiver that has ideal CSI. The performance gap between these two receivers increases slightly when the fading rate is at $f_{D} T_{s}=0.01$. At the FER $=10^{-2}$ the proposed PSP receiver is about $2 \mathrm{~dB}$ worse than the ML receiver with ideal CSI.

To demonstrate the effects of the spatial correlation on the performance of a receiver, we also examine the performance of the PSP receiver that does not take into account the spatial correlation in the fading channels (i.e., assumes spatially independent fading channels). As shown in Fig. 4, at the fading rate of $f_{D} T_{s}=0.001$ the performance of this PSP receiver is only slightly lower than the performance of the proposed PSP receiver. However at the fading rate of $f_{D} T_{s}=0.01$, the 


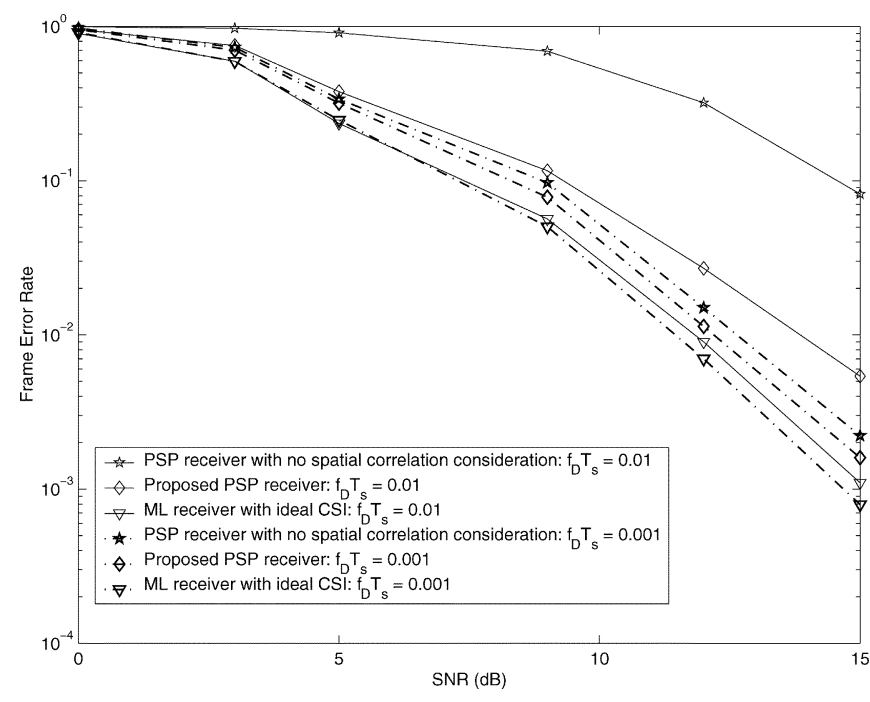

Fig. 4. Receivers' performance at the fading rate of $f_{D} T_{s}=0.001$ and $f_{D} T_{s}=0.01$

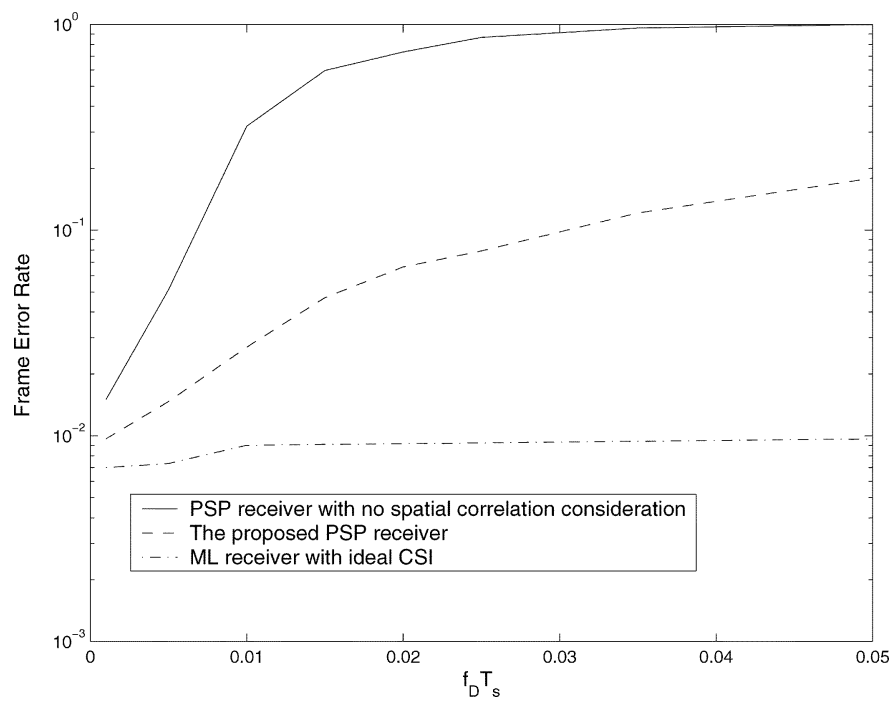

Fig. 5. Receivers' performance for different fading rate.

spatial correlation in the fading channels significantly degrades the performance of this receiver. This suggests that under fast time-varying environments (i.e., large $f_{D} T_{s}$ ), it is very important for the receiver to account for such spatial correlation in the channels. In Fig. 5, we fix the SNR at $12 \mathrm{~dB}$ and plot the FER for different fading rate $f_{D} T_{s}$. The results show that the perfor- mance of the PSP receiver that does not account for the spatial correlation degrades at a much faster rate than the proposed PSP receiver when $f_{D} T_{s}$ increases.

\section{CONCLUSION}

A PSP based receiver for joint space-time trellis decoding and channel estimation in spatially correlated time-varying fading channels is proposed in this letter. The physical channel model of the wireless fading channel with multiple transmit and multiple receive antennas is approximated by a statistical channel model. This statistical channel model permits Kalman filtering to be used for tracking the channel variations. Simulations have suggested that the proposed PSP receiver performs close to the ML receiver with ideal CSI. These simulations also confirm the importance for the receiver to account for the spatial correlation in the channel.

\section{REFERENCES}

[1] C. Cozzo and B. L. Hughes, "Joint channel estimation and data symbol detection in space-time communications," in Proc. Int. Conf. Communications, New Orleans, LA, June 18-22, 2000, pp. 287-291.

[2] Z. Liu, X. Ma, and G. Giannakis, "Space-time coding and Kalman filtering for time-selective fading channels," IEEE Trans. Commun., vol. 50, pp. 183-186, Feb. 2002.

[3] Y. Xue and X. Zhu, "PSP decoder for space-time Trellis code based on accelerated self-tuning LMS algorithm," Electron. Lett., vol. 36, no. 17, pp. 1472-1474, Aug. 2000.

[4] C. Cozzo and B. L. Hughes, "An adaptive receiver for space-time Trellis codes based on per-survivor processing," IEEE Trans. Commun., vol. 50, pp. 1213-1216, Aug. 2002.

[5] A. Abdi and M. Kaveh, "A space-time correlation model for multielement antenna systems in mobile fading channels," IEEE J. Select. Areas Commun., vol. 20, pp. 550-560, Apr. 2002.

[6] V. K. Nguyen, L. B. White, E. Jaffrot, M. Soamiadana, and I. Fijalkow, "Recursive receiver structures for general diversity channels with time correlated flat fading," IEEE J. Select. Areas Commun., vol. 21, pp. 754-764, June 2003.

[7] H. S. Wang and N. Moayeri, "Finite-state Markov channel-A useful model for radio communication channels," IEEE Trans. Veh. Technol., vol. 44, pp. 163-171, Feb. 1995.

[8] Q. Dai and E. Shwedyk, "Detection of bandlimited signals over frequency selective Rayleigh fading channels," IEEE Trans. Commun., vol. 42, pp. 941-950, Feb./Mar./Apr. 1994.

[9] M. K. Tsatsanis, G. B. Giannakis, and G. Zhou, "Estimation and equalization of fading channels with random coefficients," Signal Process., vol. 53, pp. 211-229, 1996.

[10] R. Raheli, A. Polydoros, and C.-K. Tzou, "Per-Survivor processing: A general approach to MLSE in uncertain environments," IEEE Trans. Commun., vol. 43, pp. 354-364, Feb./Mar./Apr. 1995.

[11] V. Tarokh, N. Seshadri, and A. R. Calderbank, "Space-time codes for high data rate wireless communication: Performance criterion and code construction," IEEE Trans. Inform. Theory, vol. 44, pp. 744-764, Mar. 1998. 